\title{
Synthesis and Characterization of Long-chain Branched Poly(ether imide)s with $\mathbf{A}_{3}$ Comonomers
}

Joseph M. Dennis*, Ryan J. Mondschein*, Josh D. Wolfgang*, Maruti Hegde*, Roy Odle”, and Timothy E. Long*

*Department of Chemistry, Macromolecules Innovation Institute

Virginia Tech, Blacksburg, VA 24061

${ }^{\#}$ SABIC, Mt Vernon, IN 47620

*Corresponding author's email address: telong@,vt.edu 


\section{Supporting Information}

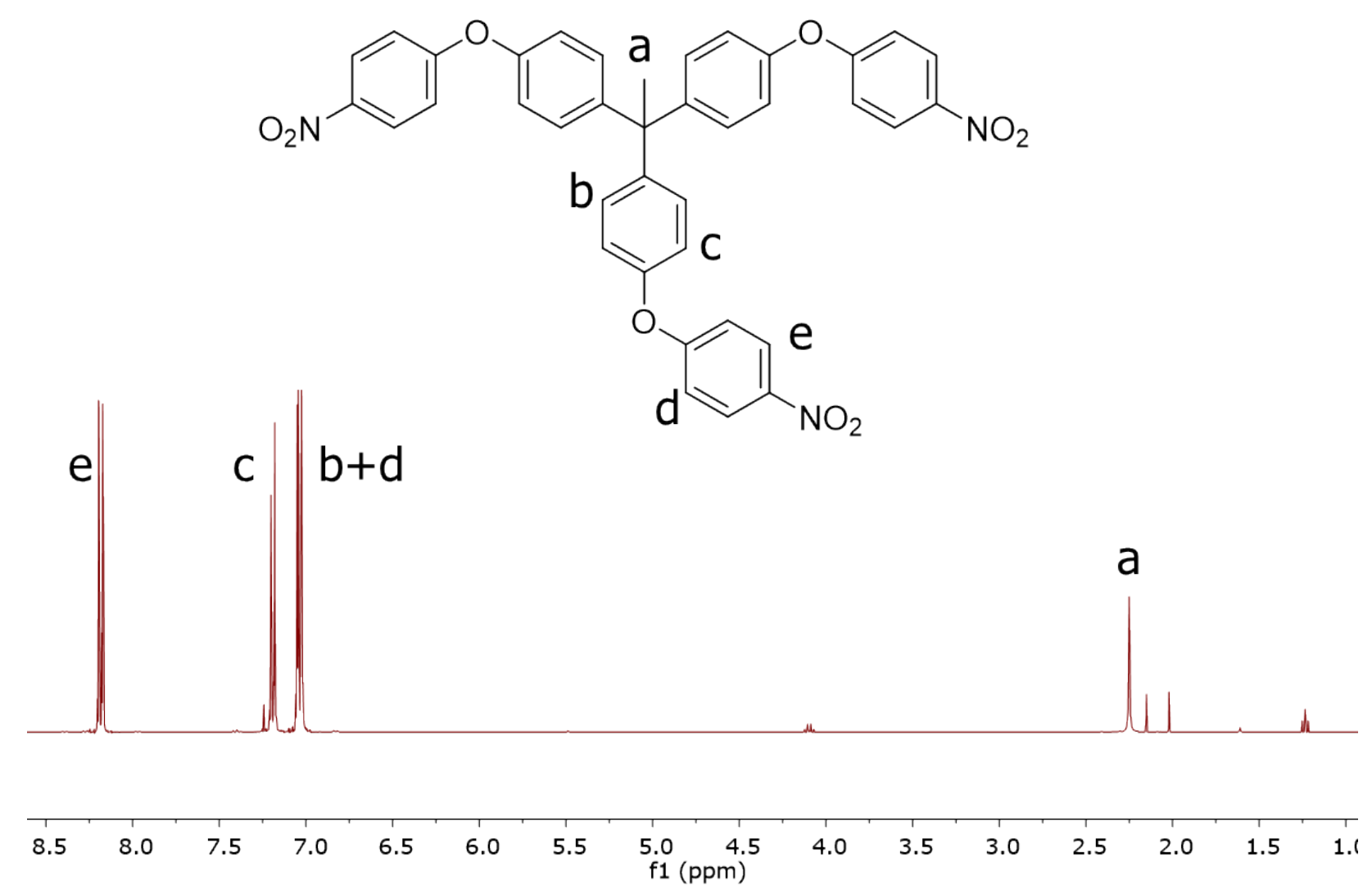

Figure S1: ${ }^{1} \mathrm{H}$ NMR spectroscopy of tris((p-nitrophenoxy)phenyl) ethane. 


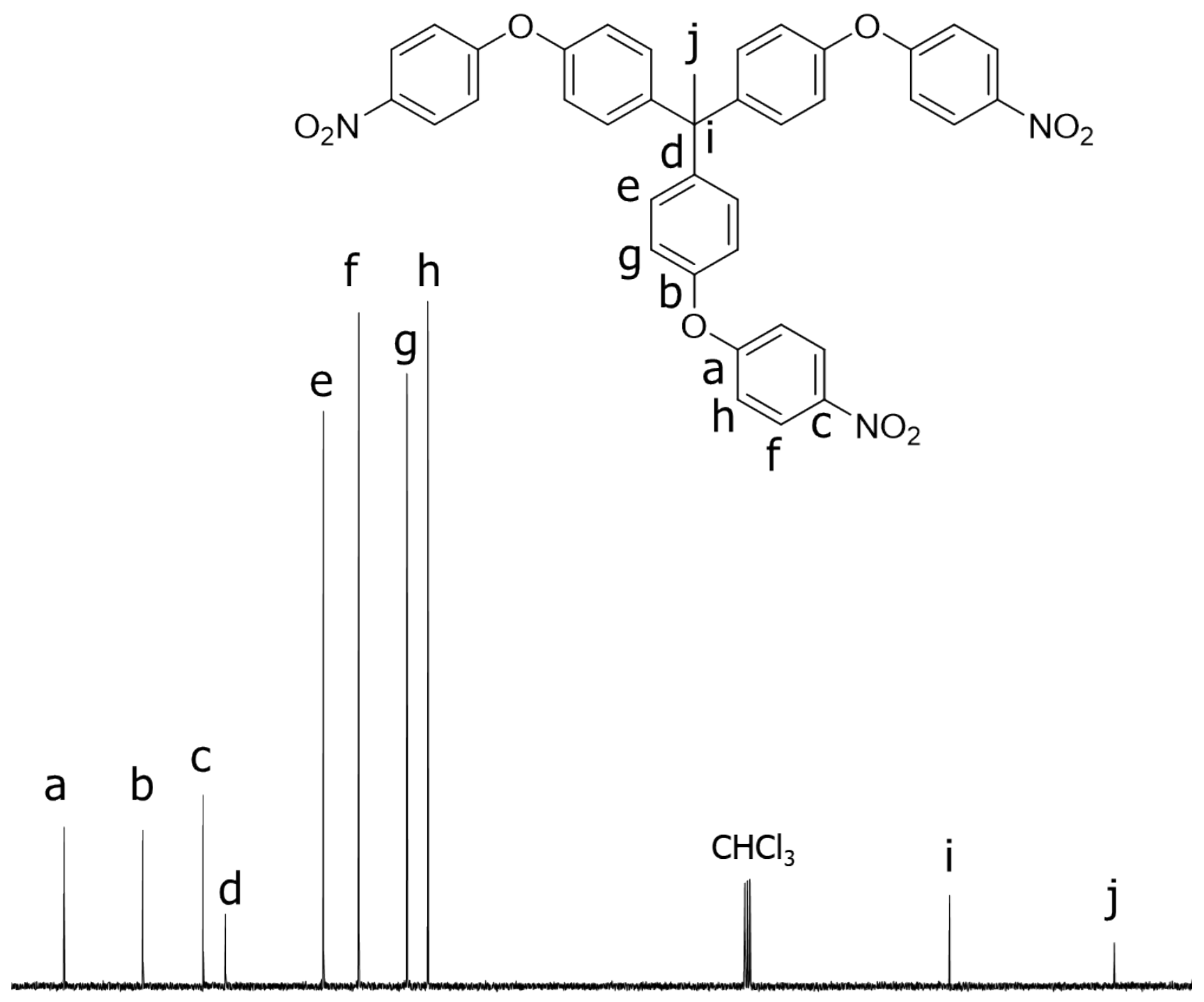

Figure S2: ${ }^{13} \mathrm{C}$ NMR spectroscopy of tris((p-nitrophenoxy)phenyl) ethane. 

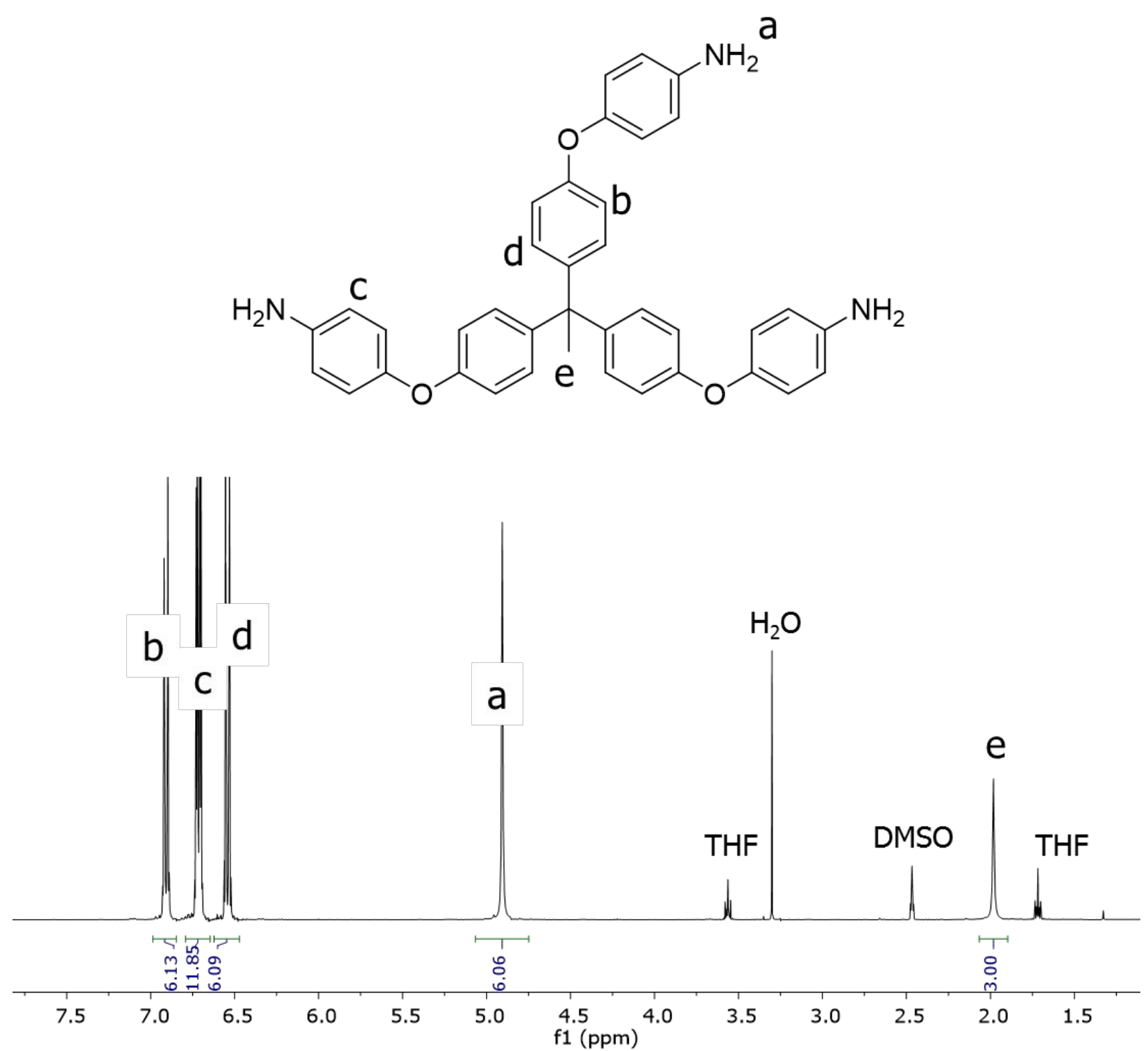

Figure S3: ${ }^{1} \mathrm{H}$ NMR spectroscopy of tris((p-aminophenoxy)phenyl) ethane (TAPE). 


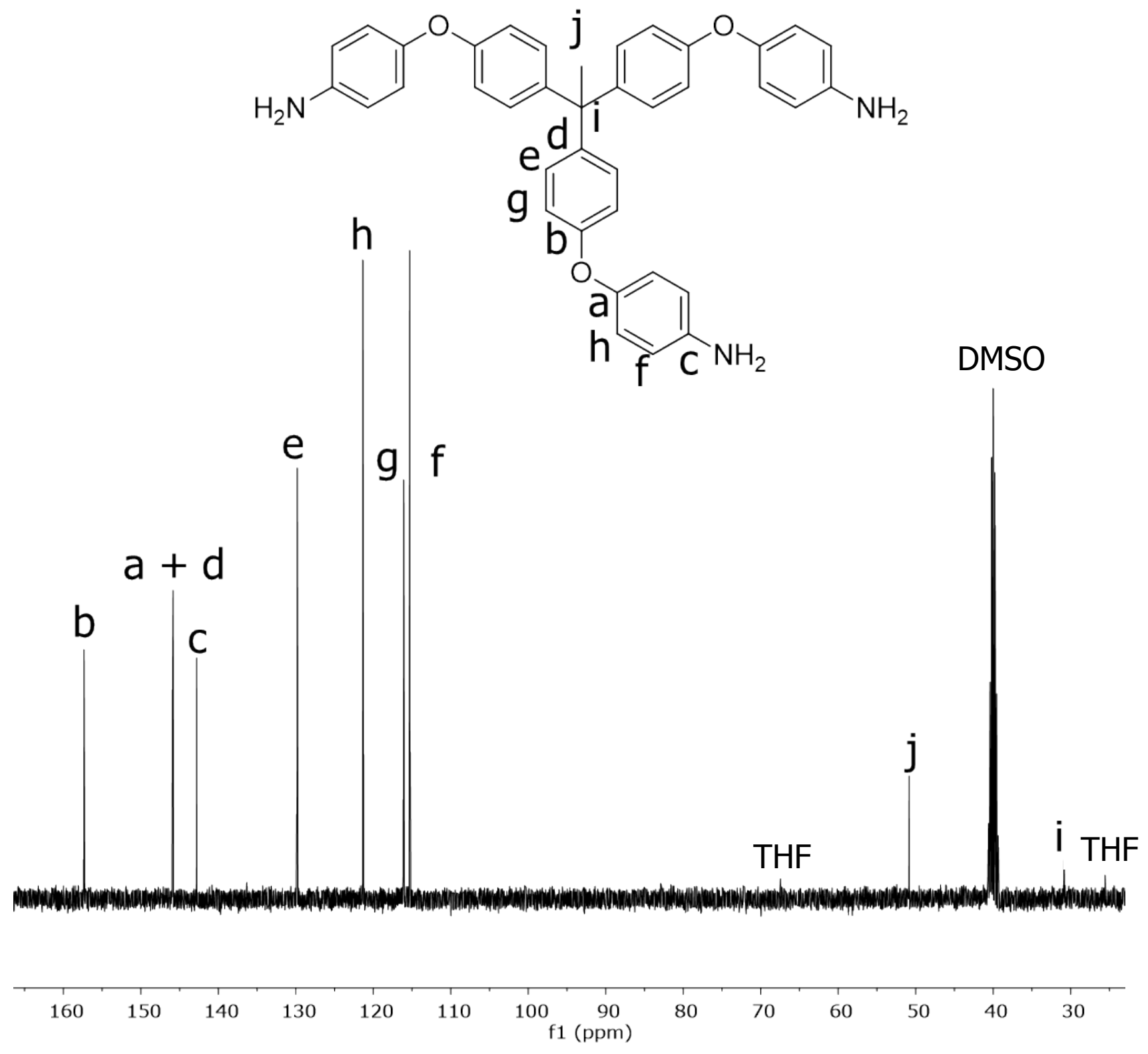

Figure S4: ${ }^{13} \mathrm{C}$ NMR spectroscopy of tris((p-aminophenoxy)phenyl) ethane. 


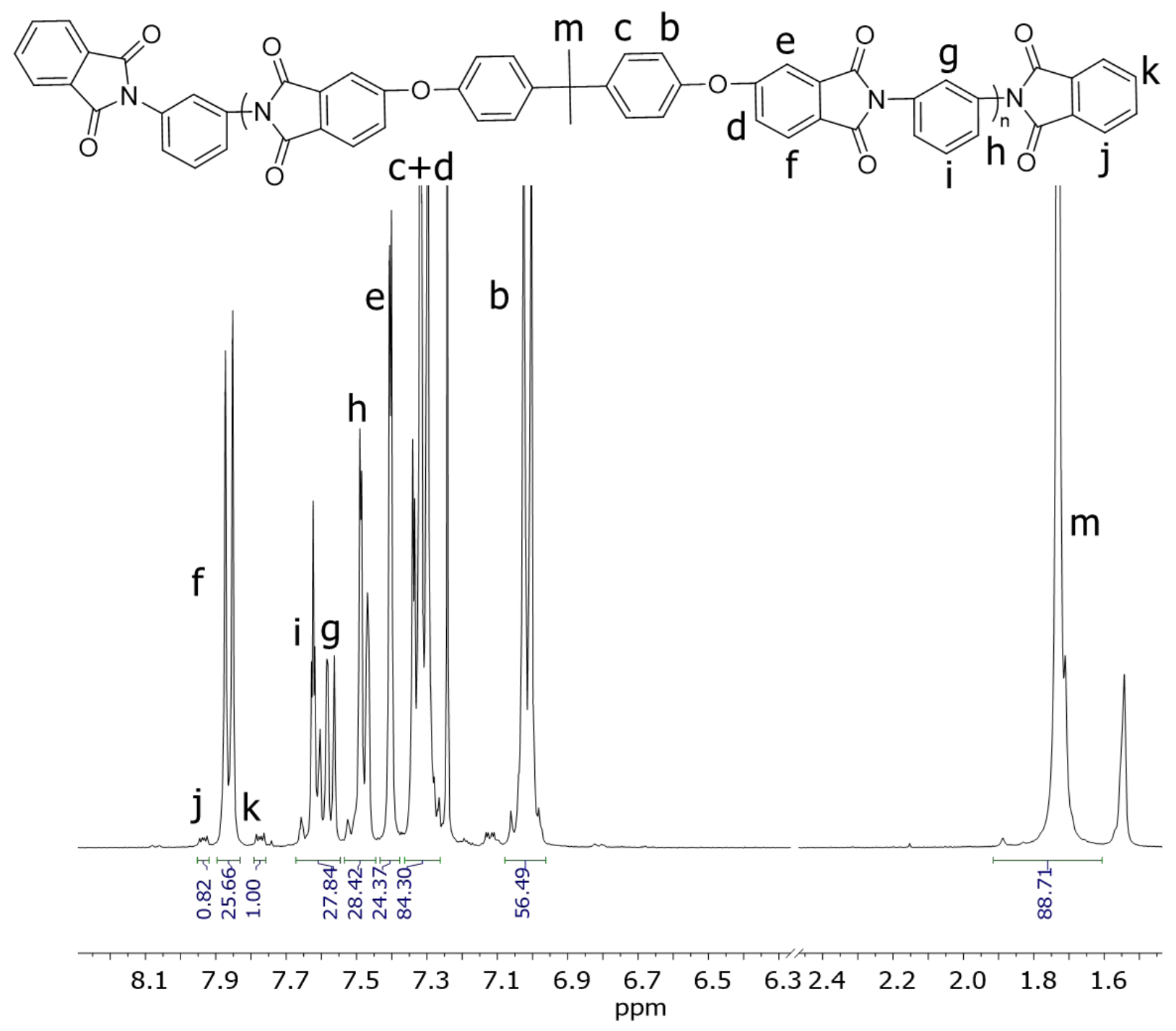

Figure S5: ${ }^{1} \mathrm{H}$ NMR spectroscopy of linear poly(ether imide) with endgroup protons indicated. 
Table S1: Summary of tensile properties of LCB-PEIs following ASTM D638.

\begin{tabular}{|c|c|c|c|c|c|c|c|c|c|c|c|c|c|}
\hline 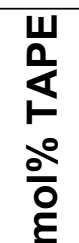 & 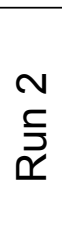 & 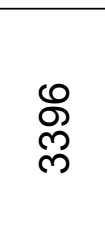 & $\stackrel{N}{\mp}$ & 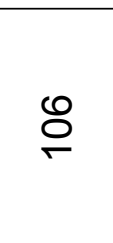 & 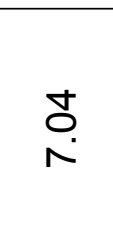 & の & 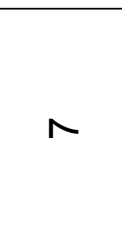 & $\sigma$ & $\stackrel{\circ}{\circ}$ & $\Lambda$ & $\begin{array}{l}\text { Oे } \\
\text { Oִ }\end{array}$ & $m$ & $\sim$ \\
\hline $\begin{array}{l}\frac{\bar{m}}{\tilde{u}} \\
\text { r } \\
\text { m }\end{array}$ & 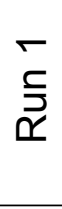 & $\begin{array}{l}\text { Ð్ } \\
\text { लె }\end{array}$ & $\stackrel{\curvearrowright}{\mp}$ & శั & $\stackrel{8}{\circ}$ & $\stackrel{ }{-}$ & $\infty$ & $\hat{\sim}$ & $\stackrel{\circ}{\circ}$ & 으 & סo & $\sim$ & $r$ \\
\hline $\begin{array}{l}\text { U. } \\
\frac{\alpha}{\digamma} \\
\frac{0}{0}\end{array}$ & 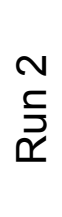 & ণ্লি & $\stackrel{\Sigma}{\Sigma}$ & ๑ே & ○ & $\stackrel{ }{\sim}$ & $\infty$ & $\stackrel{\circ}{\square}$ & $\stackrel{\circ}{\circ}$ & 으 & $\begin{array}{l}\text { Õ } \\
\text { Oִ }\end{array}$ & $N$ & $r$ \\
\hline 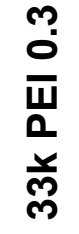 & 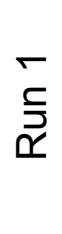 & 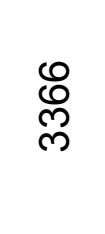 & $\stackrel{ }{F}$ & ది & ָ̊ & $\mp$ & $\infty$ & $\stackrel{\sim}{N}$ & $\stackrel{0}{\circ}$ & 으 & రo & 10 & $\sim$ \\
\hline 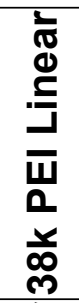 & & $\begin{array}{l}\text { N } \\
\text { m } \\
\text { m }\end{array}$ & $\stackrel{\curvearrowright}{\mp}$ & $\infty$ & $\begin{array}{l}\infty \\
\stackrel{0}{0}\end{array}$ & $\stackrel{N}{N}$ & $\hat{n}$ & m & $\stackrel{\circ}{\circ}$ & $\sim$ & Oֶ. & ిొ & $\stackrel{12}{\leftarrow}$ \\
\hline 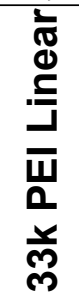 & & $\begin{array}{l}\stackrel{\infty}{\infty} \\
\text { mె }\end{array}$ & $\stackrel{\oplus}{\leftarrow}$ & ৪ & 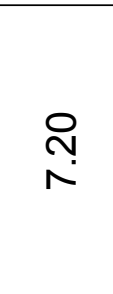 & เூ & $\stackrel{\infty}{+}$ & $\stackrel{\bullet}{\circ}$ & مْ & $r$ & రo & $\stackrel{N}{\leftarrow}$ & $\Lambda$ \\
\hline & & $\sum^{\pi}$ & $\stackrel{0}{\Sigma}$ & $\frac{\pi}{\Sigma}$ & ১0 & $\alpha^{0}$ & $\alpha^{0}$ & $\stackrel{\sqrt[\pi]{2}}{\Sigma}$ & $\frac{\pi}{\Sigma}$ & $\stackrel{0}{\Sigma}$ & ১0 & $\alpha^{\circ}$ & $\overbrace{}^{0}$ \\
\hline & 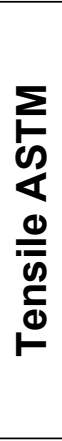 & 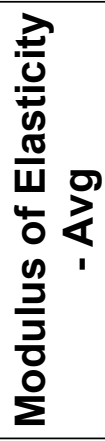 & 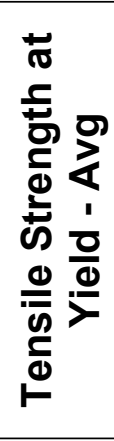 & 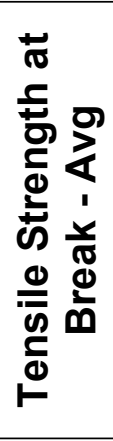 & 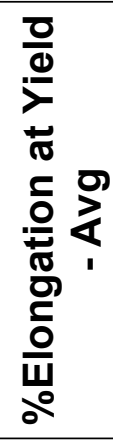 & 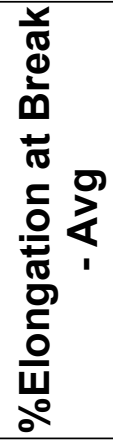 & 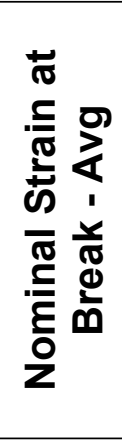 & 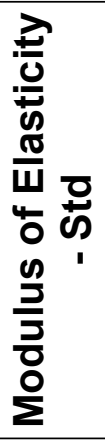 & 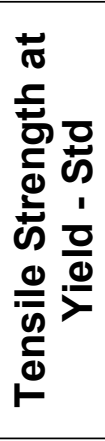 & 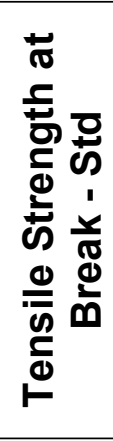 & 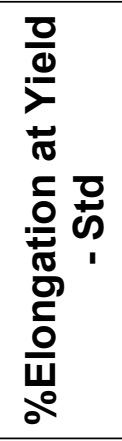 & 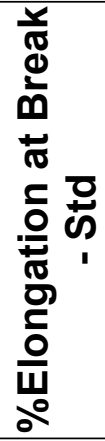 & 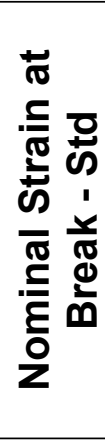 \\
\hline
\end{tabular}


Table S2: Molecular weights determined for LCB-PEIs used in mechanical analysis.

\begin{tabular}{|l|c|c|c|}
\hline Materials & $\begin{array}{c}\text { Absolute Mw } \\
\text { (g/mol) }\end{array}$ & $\begin{array}{c}\text { Absolute } \mathbf{M n} \\
\mathbf{( g / m o l})\end{array}$ & PDI \\
\hline PEI-38k & 41400 & 18900 & 2.19 \\
\hline PEI-33k & 33500 & 16700 & 2.00 \\
\hline LCB-PEI-1 & 38700 & 15500 & 2.50 \\
\hline LCB-PEI-2 & 37000 & 15100 & 2.45 \\
\hline LCB-PEI-3 & 41200 & 13900 & 2.96 \\
\hline LCB-PEI-4 & 39200 & 13300 & 2.96 \\
\hline
\end{tabular}

